\title{
MAPPINGS WHICH PRESERVE REGULAR DODECAHEDRONS
}

\author{
BYUNGBAE KIM
}

Received 4 June 2006; Accepted 5 July 2006

We prove that if a one-to-one mapping $f: \mathbb{R}^{3} \rightarrow \mathbb{R}^{3}$ preserves regular dodecahedrons, then $f$ is a linear isometry up to translation.

Copyright (c) 2006 Byungbae Kim. This is an open access article distributed under the Creative Commons Attribution License, which permits unrestricted use, distribution, and reproduction in any medium, provided the original work is properly cited.

\section{Introduction}

Let us begin with the properties of an isometry. For normed spaces $X$ and $Y$, a mapping $f: X \rightarrow Y$ is called an isometry if $f$ satisfies the equality

$$
\|f(x)-f(y)\|=\|x-y\|
$$

for all $x, y \in X$. A distance $r>0$ is said to be preserved by a mapping $f: X \rightarrow Y$ if $\| f(x)-$ $f(y) \|=r$ for all $x, y \in X$ whenever $\|x-y\|=r$.

If $f$ is an isometry, then every distance $r>0$ is preserved by $f$, and conversely. We can now raise a question whether each mapping that preserves certain distances is an isometry. Indeed, Aleksandrov [1] had raised a question whether a mapping $f: X \rightarrow X$ preserving a distance $r>0$ is an isometry, which is now known to us as the Aleksandrov problem.

Beckman and Quarles [2] solved the Aleksandrov problem for finite-dimensional real Euclidean spaces $X=\mathbb{R}^{n}$ (see also [3-6, 10, 12-17]).

Theorem 1.1 (Beckman and Quarles). If a mapping $f: \mathbb{R}^{n} \rightarrow \mathbb{R}^{n}(2 \leq n<\infty)$ preserves a distance $r>0$, then $f$ is a linear isometry up to translation.

It is an interesting question whether the "distance $r>0$ " in the above theorem can be replaced by some properties characterized by "geometrical figures" without loss of its validity. 


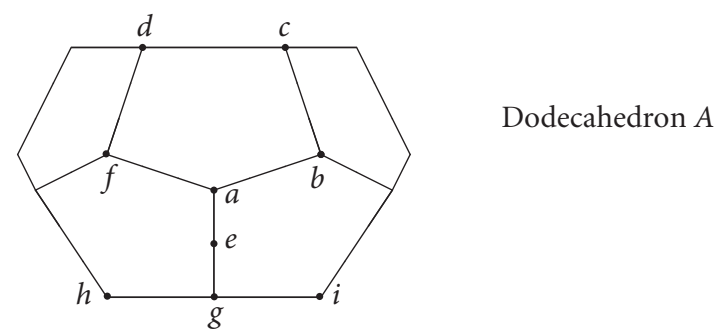

Figure 2.1

In [7-9], the authors proved that if a one-to-one mapping $f: \mathbb{R}^{n} \rightarrow \mathbb{R}^{n}$ maps every unit circle (or unit sphere, tetrahedron) onto a unit circle (or a unit sphere, tetrahedron, resp.), then $f$ is a linear isometry up to translation.

In this connection, we will extend these results to the more general three-dimensional objects, that is, we prove in this note that if a one-to-one mapping $f: \mathbb{R}^{3} \rightarrow \mathbb{R}^{3}$ maps every regular dodecahedron onto a regular dodecahedron, then $f$ is a linear isometry up to translation.

\section{Main theorem}

In the following, by a regular dodecahedron we mean a regular dodecahedron with its side length one. We first make our terms precise as follows. In Figure 2.1, we will call the point $a$ a "vertex" and the line $\overline{a b}$ an "edge" and the plane bounded by the five edges $\overline{a b}$, $\overline{b c}, \overline{c d}, \overline{d f}, \overline{f a}$ "face $a b c d f$ " or simply a "face." Further by a dodecahedron we will mean the 12 faces only and not the three-dimensional open set bounded by those 12 faces. Let us denote the three-dimensional open set bounded by the dodecahedron $A$ as "Inside of $A$ " or simply as Ins $A$.

Now we begin with the following lemma.

Lemma 2.1. Suppose that a one-to-one mapping $f: \mathbb{R}^{3} \rightarrow \mathbb{R}^{3}$ maps every dodecahedron onto a dodecahedron. Then, for any dodecahedrons $A$ and $B$, if Ins $A \cap \operatorname{Ins} B=\phi$, Ins $f(A) \cap$ Ins $f(B)=\phi$.

Proof. First, we show that if $q \notin \operatorname{Ins} A$, then $f(q) \notin \operatorname{Ins} f(A)$. In other words, we show that if $f(q) \in \operatorname{Ins} f(A)$, then $q \in \operatorname{Ins} A$. Suppose that $q \in A$. Then $f(q) \in f(A)$ and so $f(q) \notin \operatorname{Ins} f(A)$. Suppose that $q \notin \operatorname{Ins} A$ and $q \notin A$. Then choose another dodecahedron $B$ such that $q \in B$ and $B \cap A=\phi$. Then $f(B) \cap f(A)=\phi$ and therefore $f(q) \notin \operatorname{Ins} f(A)$.

Suppose now that Ins $f(A) \cap \operatorname{Ins} f(B) \neq \phi$. Then Ins $f(A) \cap f(B) \neq \phi$, which means that for some $b \in B, f(b) \in \operatorname{Ins} f(A)$. Therefore $b \in \operatorname{Ins} A$ and (Ins $A) \cap B \neq \phi$ by which we conclude that $\operatorname{Ins} A \cap \operatorname{Ins} B \neq \phi$.

We show now that if any one-to-one mapping preserves regular dodecahedrons, then it is actually an isometry. More precisely, we have the following.

TheOREM 2.2. If a one-to-one mapping $f: \mathbb{R}^{3} \rightarrow \mathbb{R}^{3}$ maps every regular dodecahedron onto a regular dodecahedron, then $f$ is a linear isometry up to translation. 


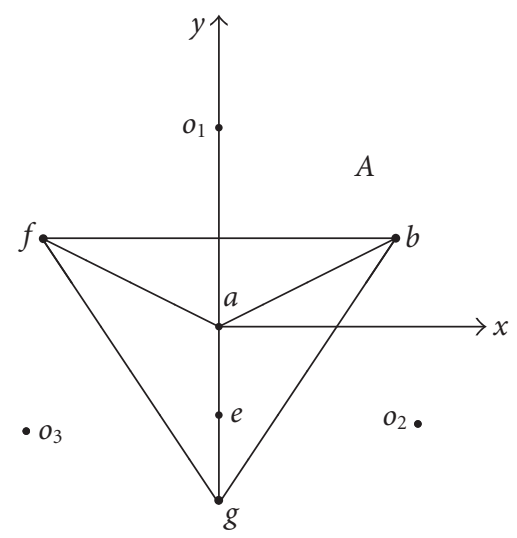

$$
\begin{aligned}
& a=(0,0,0) \\
& b=\left(\sin 54^{\circ}, \frac{1}{\sqrt{3}} \sin 54^{\circ},-z_{o}\right) \\
& f=\left(-\sin 54^{\circ}, \frac{1}{\sqrt{3}} \sin 54^{\circ},-z_{o}\right) \\
& g=\left(0,-\frac{2}{\sqrt{3}} \sin 54^{\circ},-z_{o}\right) \\
& e=\left(0,-\frac{1}{\sqrt{3}} \sin 54^{\circ},-\frac{1}{2} z_{o}\right) \\
& z_{o}=\sqrt{1-\frac{4}{3} \sin ^{2} 54^{\circ}}
\end{aligned}
$$

Figure 2.2

Proof. We show $f$ preserves the distance 1. Then the theorem of Beckman and Quarles implies that $f$ is an isometry.

We will use solid angle arguments. Let $A$ be a regular dodecahedron. For any $p \in A$, let us denote the solid angle that Ins $A$ subtends with respect to $p \in A$ as $\Omega(A, p)$. We first find the solid angles at a vertex and at an edge point. To do so, let us choose suitable coordinate axes so that the vertex $a$ of Figure 2.1 is located at the origin (see Figure 2.2). To get the coordinate of $b$, first we note that the $\angle f a b$ is a part of a regular pentagon $a b c d f$ with side length one and therefore it is $108^{\circ}$. Therefore the length of $\overline{b f}$ is $2 \sin 54^{\circ}$ and the triangle $b f g$ is a regular triangle with side length $2 \sin 54^{\circ}$. Then the $x$ coordinate of $b$ is $\sin 54^{\circ}$, the $y$ coordinate is $(1 / 3) \times \sqrt{3} \sin 54^{\circ}$, and we can find the $z$ coordinate $-z_{o}$ with the condition that the length of $\overline{a b}$ is one. Therefore $z_{o}=\sqrt{1-(4 / 3) \sin ^{2} 54^{\circ}}$. Let $e=(1 / 2) g$ which belongs to an edge of $A$. We find $\Omega(A, e)$ first.

Numerically these points are $b=(0.809,0.467,-0.357), f=(-0.809,0.467,-0.357)$, $g=(0,-0.934,-0.357), e=(0,-0.467,-0.1784), z_{o}=0.357$.

If we call $C_{1}$ the pentagon $a b c d f$, then the radius $r$ of the circle which circumscribes $C_{1}$ satisfies $2 r \cos 54^{\circ}=1$ and therefore $r=1 / 2 \cos 54^{\circ}=0.851$. Now the center $o_{1}$ of $C_{1}$ is the distance $r=0.851$ away from the origin $a$ and located along the direction of $(\overrightarrow{a b}+\overrightarrow{a f}) /|\overrightarrow{a b}+\overrightarrow{a f}|=\left(0,(1 / \sqrt{3}) \tan 54^{\circ},-z_{o} / \cos 54^{\circ}\right)$. Therefore $o_{1}=r((\overrightarrow{a b}+\overrightarrow{a f}) / \mid \overrightarrow{a b}+$ $\overrightarrow{a f} \mid)=(0,0.676,-0.517)$. Similarly the center $o_{2}$ of the pentagon $C_{2}$ which contains the vertices $g, a, b$ is located at $o_{2}=r((\overrightarrow{a b}+\overrightarrow{a g}) /|\overrightarrow{a b}+\overrightarrow{a g}|)=(0.586,-0.338,-0.517)$ and similarly $o_{3}=r((\overrightarrow{a g}+\overrightarrow{a f}) /|\overrightarrow{a g}+\overrightarrow{a f}|)=(-0.586,-0.338,-0.517)$. The two pentagons $C_{2}$ and $C_{3}$ meet at the edge $\overline{a g}$ and the vectors $e_{o_{2}}$ and $\overrightarrow{e O}_{3}$ are both perpendicular to $\overline{a g}$. Therefore, to find the (ordinary) angle between the two planes $C_{2}$ and $C_{3}$, we have only to find the angle between the two vectors $e \vec{o}_{2}$ and $e \vec{o}_{3}$. Since $e \vec{o}_{2}=a \vec{o}_{2}-\overrightarrow{a e}=a \vec{o}_{2}-(1 / 2) \overrightarrow{a g}$ and $e \vec{o}_{3}=a \vec{o}_{3}-\overrightarrow{a e}=a \vec{o}_{3}-(1 / 2) \overrightarrow{a g}$, the angle $\theta$ between these two vectors is given by

$$
\cos \theta=\frac{e \vec{o}_{2} \cdot e \vec{o}_{3}}{\left|e \vec{o}_{2}\right|\left|e \vec{o}_{3}\right|}=-0.4468
$$


and we get $\theta=116.5^{\circ}$ or $2.034 \mathrm{rad}$. This angle is often called the dihedral angle of the dodecahedron. Now we are ready to find the solid angle $\Omega(A, e)$. We use the unit of the solid angle such that the solid angle of the whole sphere with respect to its center is $4 \pi$. Then the solid angle that Ins $A$ subtends with respect to $e \in A$ is

$$
\Omega(A, e)=4 \pi \frac{\theta}{2 \pi}=2 \theta=4.068 \equiv \Omega_{e}
$$

Having found the solid angle $\Omega_{e}$ at the edge point of the regular dodecahedron, let us now find the solid angle that Ins $A$ subtends at the vertex $a$. For that we use the result of [11], where it is shown that given any three vectors $\mathbf{T}_{1}, \mathbf{T}_{2}, \mathbf{T}_{3}$, all starting from the origin, the solid angle $\Omega$ that these three vectors subtend with respect to the origin (i.e., the solid angle that the (not necessarily regular) tetrahedron whose vertices are the end tips of these three vectors and the origin subtends with respect to the origin) is

$$
\tan \left(\frac{1}{2} \Omega\right)=\frac{\left[\mathbf{T}_{1} \mathbf{T}_{2} \mathbf{T}_{3}\right]}{T_{1} T_{2} T_{3}+\left(\mathbf{T}_{1} \cdot \mathbf{T}_{2}\right) T_{3}+\left(\mathbf{T}_{2} \cdot \mathbf{T}_{3}\right) T_{1}+\left(\mathbf{T}_{3} \cdot \mathbf{T}_{1}\right) T_{2}}
$$

Here $\left[\mathbf{T}_{1} \mathbf{T}_{2} \mathbf{T}_{3}\right] \equiv\left|\mathbf{T}_{1} \cdot \mathbf{T}_{2} \times \mathbf{T}_{3}\right|$ and $T_{i}=\left|\mathbf{T}_{i}\right|(i=1,2,3)$. Note that this expression does not depend on the lengths of the three vectors. For example, when $\mathbf{T}_{1}, \mathbf{T}_{2}, \mathbf{T}_{3}$ are mutually orthogonal, we get $\Omega=\pi / 2$.

Now we are ready to find the solid angle that Ins $A$ subtends at the vertex $a, \Omega(A, a)$. To find $\Omega$, let $\mathbf{T}_{1}=\overrightarrow{a g}, \mathbf{T}_{2}=\overrightarrow{a f}$, and $\mathbf{T}_{3}=\overrightarrow{a b}$. Then using (2.3) we can compute the solid angle we want. We find $\left[\mathbf{T}_{1} \mathbf{T}_{2} \mathbf{T}_{3}\right]=0.809, \mathbf{T}_{1} \cdot \mathbf{T}_{2}=\mathbf{T}_{2} \cdot \mathbf{T}_{3}=\mathbf{T}_{3} \cdot \mathbf{T}_{1}=-0.309$, and $T_{1}=$ $T_{2}=T_{3}=1$. Therefore we get

$$
\begin{gathered}
\Omega=2 \tan ^{-1}\left(\frac{0.809}{1-3 \times 0.309}\right)=2 \tan ^{-1} 11.082, \\
\Omega(A, a)=2 \tan ^{-1} 11.082=2.962 \equiv \Omega_{v} .
\end{gathered}
$$

Let us summarize our results above. Suppose that $p \in A$ where $p$ is a point and $A$ is a regular dodecahedron. If $p$ is a vertex, say $p=a$, then the solid angle that Ins $A$ subtends with respect to $p$ is $\Omega(A, p)=\Omega_{v}=2.962$. If $p$ is a point which belongs to an edge and is not a vertex, then $\Omega(A, p)=\Omega_{e}=4$.068. If $p \in A$ is neither a vertex nor an edge point, then $\Omega(A, p)=2 \pi$.

Suppose now that $a$ is a vertex of a regular dodecahedron $A=A_{1}$. We show then that $f(a)$ is a vertex of $f(A)$ too. Or we have only to show that $\Omega(f(A), f(a))=\Omega_{v}$. Construct three more regular dodecahedrons $A_{i}(i=2,3,4)$ such that $a$ is the common vertex of all four regular dodecahedrons $A_{i}(i=1, \ldots, 4)$ and Ins $A_{i} \cap \operatorname{Ins} A_{j}=\phi$ for any $i \neq j$ (see the appendix). Then $f(a)$ belongs to $f\left(A_{i}\right)$ for $i=1, \ldots, 4$ and by the above lemma Ins $f\left(A_{i}\right) \cap$ Ins $f\left(A_{j}\right)=\phi$ for $i \neq j$. Now the solid angle that Ins $f\left(A_{i}\right)$ subtends with respect to $f(a)$ 
can be $\Omega_{v}, \Omega_{e}$, or $2 \pi$. Further

$$
\sum_{i=1}^{4} \Omega\left(f\left(A_{i}\right), f(a)\right) \leq 4 \pi .
$$

Since

$$
3 \Omega_{v}+\Omega_{e}=3 \times(2.962)+4.068=12.954>4 \pi=12.566,
$$

we conclude that $\Omega\left(f\left(A_{i}\right), f(a)\right)=\Omega_{v}(i=1, \ldots, 4)$ and that $f(a)$ is a vertex of $f(A)=$ $f\left(A_{1}\right)$.

Now we prove the statement of the theorem. Given any two points $a$ and $b$ which are distanced by the unit distance from each other, form two regular dodecahedrons $A$ and $B$ such that the following three conditions are met. (1) Both $a$ and $b$ are common vertices of $A$ and $B$. (2) Ins $A \cap \operatorname{Ins} B=\phi$. (3) No other vertices are common vertices of $A$ and $B$. This means that the two dodecahedrons $A$ and $B$ share exactly one edge which has end points $a$ and $b$. Now it is obvious that the two dodecahedrons $f(A)$ and $f(B)$ also satisfy the above three conditions with the common vertices $f(a)$ and $f(b)$. Therefore the distance between $f(a)$ and $f(b)$ is again one too. Since $f$ preserves the unit distance, by the theorem of Beckman and Quarles, we conclude that $f$ is a linear isometry up to translation.

\section{Appendix}

We show that we can form four regular dodecahedrons $A_{i}(i=1, \ldots, 4)$ such that $g \in A_{i}$ is a common vertex of $A_{i}(i=1, \ldots, 4)$ and Ins $A_{i} \cap \operatorname{Ins} A_{j}=\phi$ for any $i \neq j(i, j=1, \ldots, 4)$. Let $A=A_{1}$ be a regular dodecahedron as shown in Figure 2.1. Choose a coordinate such that $g$ is at the origin and the pentagon containing the vertices $h, g, i$ is contained completely in the $x z$ plane. Then $i=\left(\sin 54^{\circ}, 0,-\cos 54^{\circ}\right)$ and $h=\left(-\sin 54^{\circ}, 0,-\cos 54^{\circ}\right)$. Further $a=\left(0, \sin 54^{\circ} \sqrt{3-\tan ^{2} 54^{\circ}},-\cos 108^{\circ} / \cos 54^{\circ}\right)$. The coordinate of $a$ is uniquely determined by the condition that $a$ is in the $y z$ plane with $|\overrightarrow{g a}|=1$ and $|\overrightarrow{h a}|=2 \sin 54^{\circ}$. Numerically $g=(0,0,0) \equiv g_{1}, i=(0.809,0,-0.588) \equiv i_{1}, h=(-0.809,0,-0.588) \equiv h_{1}$, and $a=(0,0.8507,0.5257) \equiv a_{1}$.

Let $L_{1}$ be a reflection through the $x z$ plane, that is, $L_{1}(x, y, z)=(x,-y, z)$. Call $L_{1}\left(A_{1}\right)=$ $A_{2}$. Let $L_{2}$ be a $90^{\circ}$ rotation around $z$ axis followed by the reflection through the $x y$ plane, that is, $L_{2}(x, y, z)=(-y, x,-z)$. Call $L_{2}\left(A_{1}\right)=A_{3}$ and $L_{2}\left(A_{2}\right)=A_{4}$ (see Figure A.1). Note that $A_{4}=L_{2} L_{1}\left(A_{1}\right)$ and $L_{2} L_{1}(x, y, z)=(y, x,-z)$. Therefore $a_{4}=L_{2} L_{1}\left(a_{1}\right)=(0.8507,0$, $-0.5257)$ and $i_{4}=L_{2} L_{1}\left(i_{1}\right)=(0,0.809,0.588)$. We claim that Ins $A_{i} \cap \operatorname{Ins} A_{j}=\phi$ for any $i \neq j(i, j=1, \ldots, 4)$.

By symmetry we have only to check the part where $x \geq 0$ and $y \geq 0$. We think of $z$ coordinate as a height function. In Figure A.1, $g_{1,2}=g_{3,4}=(0,0,0) \equiv g$. The "slope" of $\overline{g a_{1}}$ is $a_{1 z} / a_{1 y}=0.5257 / 0.8507=0.6180$, which is smaller than the slope of $\overline{g i_{4}}$ that is $i_{4 z} / i_{4 y}=$ $0.588 / 0.809=0.7268$. Similarly, The slope of $\overline{g i_{1}}$ is $i_{1 z} / i_{1 x}=-0.588 / 0.809=-0.7268$, which is smaller than the slope of $\overline{g a_{4}}$ that is $a_{4 z} / a_{4 x}=-0.5257 / 0.8507=-0.6180$. This 

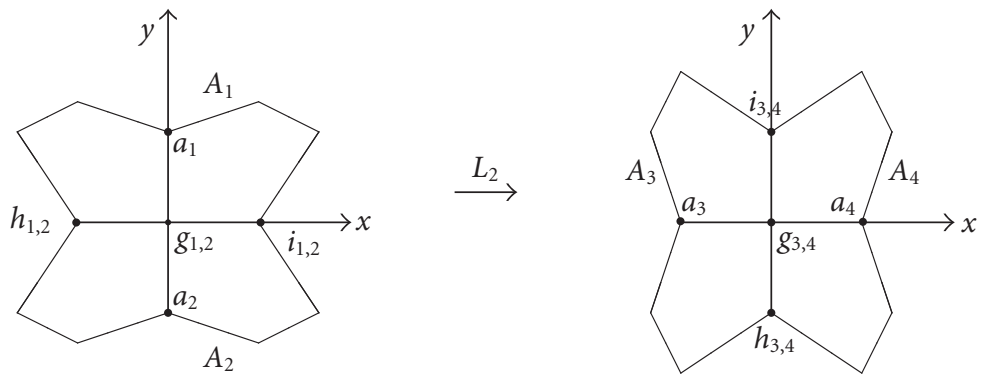

Figure A.1

means that $A_{4}$ is "higher" than $A_{1}$ except at the origin $g$. Therefore we conclude that Ins $A_{i} \cap \operatorname{Ins} A_{j}=\phi$ for any $i \neq j(i, j=1, \ldots, 4)$.

\section{Acknowledgment}

This work was done during the author's 2005-2006 sabbatical leave from Hongik University in Korea.

\section{References}

[1] A. D. Aleksandrov, Mappings of families of sets, Soviet Mathematics - Doklady 11 (1970), 116120.

[2] F. S. Beckman and D. A. Quarles Jr., On isometries of Euclidean spaces, Proceedings of the American Mathematical Society 4 (1953), no. 5, 810-815.

[3] W. Benz, Isometrien in normierten Räumen, Aequationes Mathematicae 29 (1985), no. 2-3, 204209.

[4] _ An elementary proof of the theorem of Beckman and Quarles, Elemente der Mathematik 42 (1987), no. 1, 4-9.

[5] R. L. Bishop, Characterizing motions by unit distance invariance, Mathematics Magazine 46 (1973), 148-151.

[6] D. Greenwell and P. D. Johnson, Functions that preserve unit distance, Mathematics Magazine 49 (1976), no. 2, 74-79.

[7] S.-M. Jung and B. Kim, Unit-circle-preserving mappings, International Journal of Mathematics and Mathematical Sciences 2004 (2004), no. 66, 3577-3586.

[8] _ Unit-sphere preserving mappings, Glasnik Matematički. Serija III 39(59) (2004), no. 2, 327-330.

[9] B. Kim, Mappings which preserve regular tetrahedrons, in press.

[10] B. Mielnik and Th. M. Rassias, On the Aleksandrov problem of conservative distances, Proceedings of the American Mathematical Society 116 (1992), no. 4, 1115-1118.

[11] A. Oosterom and J. Strackee, The solid angle of a plane triangle, IEEE Transactions on Biomedical Engineering 30 (1983), no. 2, 125-126.

[12] Th. M. Rassias, Is a distance one preserving mapping between metric spaces always an isometry?, The American Mathematical Monthly 90 (1983), no. 3, 200.

[13] _ Mappings that preserve unit distance, Indian Journal of Mathematics 32 (1990), no. 3, 275-278.

[14] Th. M. Rassias and P. Šemrl, On the Mazur-Ulam theorem and the Aleksandrov problem for unit distance preserving mappings, Proceedings of the American Mathematical Society 118 (1993), no. 3, 919-925. 
[15] Th. M. Rassias and C. S. Sharma, Properties of isometries, Journal of Natural Geometry 3 (1993), no. 1, 1-38.

[16] E. M. Schröder, Eine Ergänzung zum Satz von Beckman and Quarles, Aequationes Mathematicae 19 (1979), no. 1, 89-92.

[17] C. G. Townsend, Congruence-preserving mappings, Mathematics Magazine 43 (1970), 37-38.

Byungbae Kim: Mathematics Section, College of Science and Technology, Hong-Ik University, Chochiwon 339-701, South Korea

E-mail address: bkim@hongik.ac.kr 


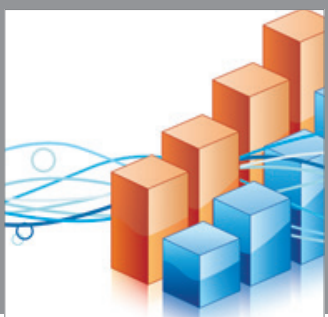

Advances in

Operations Research

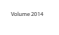

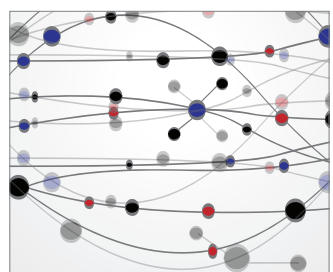

\section{The Scientific} World Journal
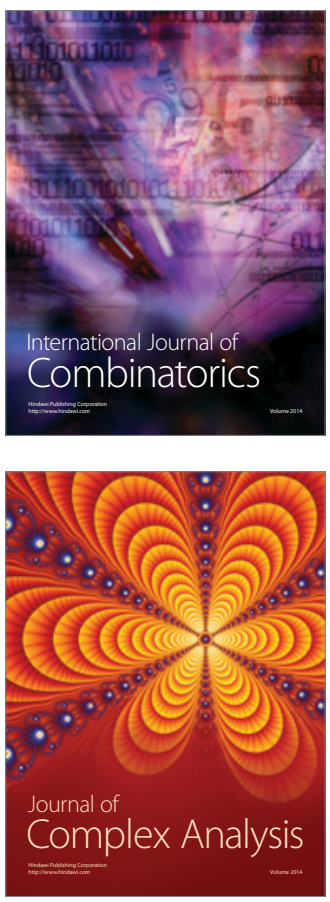

International Journal of

Mathematics and

Mathematical

Sciences
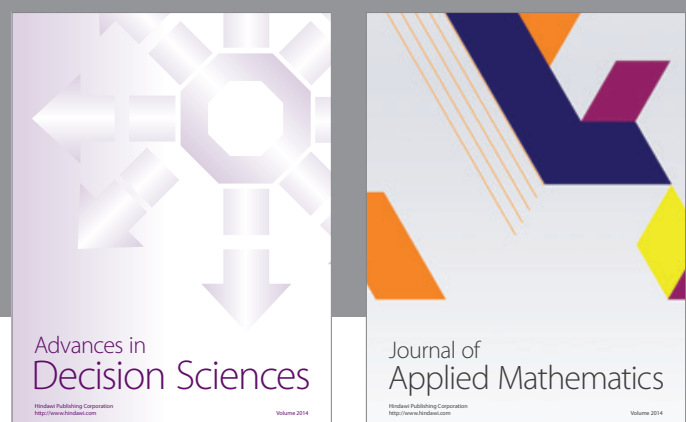

Journal of

Applied Mathematics
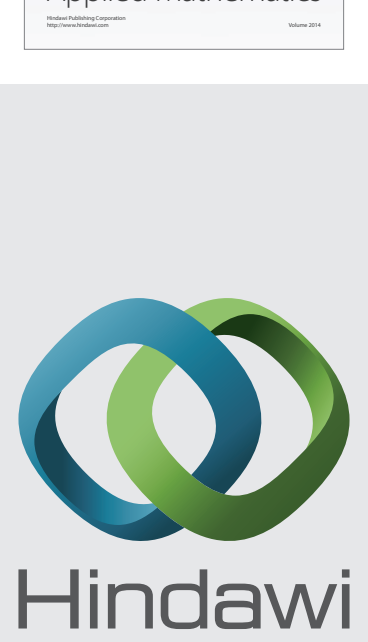

Submit your manuscripts at http://www.hindawi.com
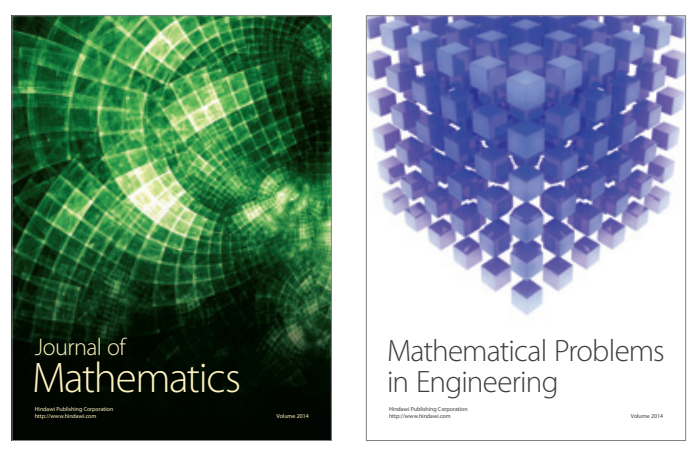

Mathematical Problems in Engineering
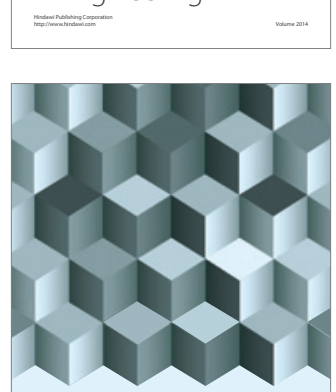

Journal of

Function Spaces
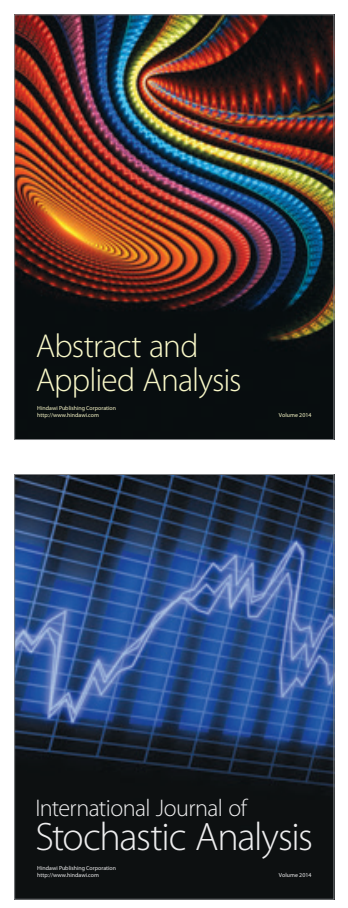

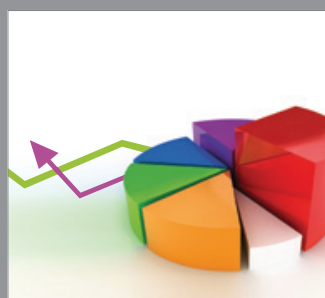

ournal of

Probability and Statistics

Promensencen
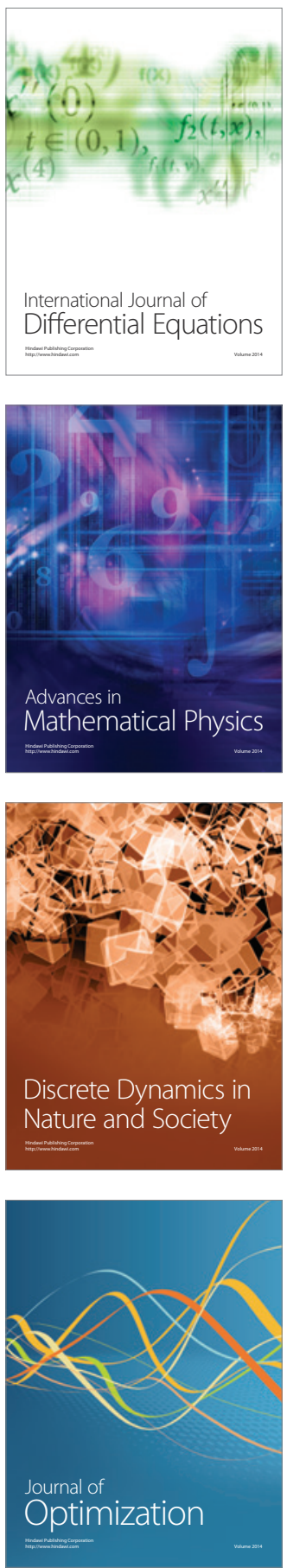